Mathematical Research Letters 1, 701-715 (1994)

\title{
SYMMETRY OF THE GINZBURG LANDAU MINIMIZER IN A DISC
}

\author{
Elliott H. Lieb AND Michael Loss
}

\begin{abstract}
A в stract. The Ginzburg-Landau energy minimization problem for a vector field on a two dimensional disc is analyzed. This is the simplest nontrivial example of a vector field minimization problem and the goal is to show that the energy minimizer has the full geometric symmetry of the problem. The standard methods that are useful for similar problems involving real valued functions cannot be applied to this situation. Our main result is that the minimizer in the class of symmetric fields is stable, i.e., the eigenvalues of the second variation operator are all nonnegative.
\end{abstract}

\section{Introduction}

There are many energy minimization problems having a geometric symmetry and for which one can show that the energy minimizer has the same symmetry as the problem itself. Typically this is done by using a rearrangement inequality of some sort. However, and this is the important point, rearrangement inequalities work (if they work at all) only when the variable is a function and not something more complicated like a vector field.

There are several important problems in which the variable is one or more vector or tensor fields and for which the minimizer is believed to be symmetric. Examples include the full multi-field Ginzburg-Landau problem for a superconductor in a magnetic field, the 't Hooft-Polyakov monopole and the Skyrme model (see [LE2] for a review). They are all unresolved. In this paper we analyze the simplest possible nontrivial example of a vector field energy minimization problem - the Ginzburg-Landau problem for a complex scalar field in a disc. It has exercised many authors (see, e.g., $[\mathrm{JT}],[\mathrm{BBH}]$ and references therein) but no one has been able to show that the obvious symmetric vector field minimizes the energy (except in the

(C)1994 by the authors. Reproduction of this article, in its entirety, by any means is permitted for non-commercial purposes.

Received October 5, 1994.

Work of E. Lieb partially supported by NSF grant PHY 90-19433 A03.

Work of M. Loss partially supported by NSF grant DMS 92-07703. 
weak coupling regime where convexity holds). In fact, it has not even been shown that the symmetric solution is stable under perturbations, and it is the purpose of this paper to prove just that. We do so by using a mixture of rearrangement inequalities on different components of the vector field and, while our methods are highly specialized to this problem, we believe that it is one of the few examples in which light can be shed on the symmetry of an energy minimizing vector field.

As an illustration of the problem in which the variable, $\psi$, is a function, one could mention the following: Let $\mathbf{B}_{n}$ denote the closed unit ball centered at $0 \in \mathbf{R}^{n}$ and let $\psi$ denote a real valued function on $\mathbf{B}_{n}$ that vanishes on $\partial \mathbf{B}_{n}$, the boundary of $\mathbf{B}_{n}$, and whose gradient is square integrable. Then we set

$$
\mathcal{F}(\psi)=\int_{\mathbf{B}_{n}}|(\nabla \psi)(x)|^{2} \mathrm{~d} x+\int_{\mathbf{B}_{n}}\left[1-\psi(x)^{2}\right]^{2} \mathrm{~d} x
$$

and seek to minimize $\mathcal{F}(\psi)$. It is well known that there is a minimizer and that it is spherically symmetric, i.e., $\psi(x)=\psi(y)$ if $|x|=|y|$. The minimizer thus retains the symmetry of the problem. Indeed, more is true: $\psi$ is symmetric decreasing, i.e., $\psi(x) \geq \psi(y)$ if $|x| \leq|y|$. While there are other methods to prove the symmetry, one of the simplest is to do so by using rearrangement inequalities to show that $\psi$ is symmetric decreasing.

The first step in this process is to observe that replacing $\psi$ by $|\psi|$ does not change $|\nabla \psi|^{2}$ and hence does not change the energy $\mathcal{F}(\psi)$. The second step is to replace $|\psi|$ by the equimeasurable function $\psi^{*}$ which is defined to be the symmetric decreasing rearrangement of $|\psi|$. Certainly $\psi^{*}$ satisfies the boundary conditions. The equimeasurability of $\psi^{*}$ and $|\psi|$ guarantees that $\int\left[1-\psi^{2}\right]^{2}=\int\left[1-\psi^{* 2}\right]^{2}$. The important inequality concerns the kinetic energy, or Dirichlet integral. It is

$$
\int_{\mathbf{B}_{n}}|\nabla| \psi||^{2} \geq \int_{\mathbf{B}_{n}}\left|\nabla \psi^{*}\right|^{2}
$$

This shows that among the energy minimizers there is at least one that is symmetric decreasing.

We now turn to the Ginzburg-Landau problem in the disc $\mathbf{D}=\mathbf{B}_{2}$ in $\mathbf{R}^{2}$, which looks deceptively similar to the above problem. For one thing the variable is now a real vector field $\psi(x)=(f(x), g(x))$ instead of a single function. It is customary to introduce the complex valued function $\phi(x)=f(x)+i g(x)$. The energy functional is

$$
\begin{aligned}
\mathcal{E}(\psi) & =\int_{\mathbf{D}}\left\{(\nabla f(x))^{2}+(\nabla g(x))^{2}+J\left(f(x)^{2}+g(x)^{2}\right)\right\} \mathrm{d} x \\
& =\int_{\mathbf{D}}\left\{|\nabla \phi|^{2}+J\left(|\phi|^{2}\right)\right\} .
\end{aligned}
$$


Usually, $J: \mathbf{R}^{+} \rightarrow \mathbf{R}^{+}$is taken to be the function $J(t)=\lambda(1-t)^{2}$ with $\lambda>0$. For our purposes we can generalize this to $J$ satisfying certain conditions, which we assume henceforth:

(i) $J(0)=\lambda>0, \quad J(1)=0, \quad J(t) \geq 0$ if $t>1$,

(ii) $J(t)$ is monotone decreasing and convex on the interval $[0,1]$,

(iii) $J$ is twice differentiable on $[0,1]$.

The gradients of $\psi$ are assumed to be square integrable and the condition on $\psi$ on the boundary of $\mathbf{D}$ is

$$
\psi(x)=x=\left(x_{1}, x_{2}\right)=(\cos \theta, \sin \theta) .
$$

We denote the class of $H^{1}(\mathbf{D})$ functions satisfying (1.4) by $\mathcal{C}$. The problem is to minimize $\mathcal{E}(\psi)$ subject to $\psi \in \mathcal{C}$.

For this problem it is a standard fact that a minimizer exists and satisfies the Euler-Lagrange pair of equations

$$
-\Delta \psi+J^{\prime}\left(\psi^{2}\right) \psi=0
$$

with $\psi^{2}=f^{2}+g^{2}$. The obvious conjecture about a minimizer $\psi$ is that it is a "hedgehog", i.e., for some nonnegative function $f$ defined on $[0,1]$ with $f(1)=1$

$$
\psi(x)=f(r)(\cos \theta, \sin \theta)
$$

where $r:=\sqrt{x_{1}^{2}+x_{2}^{2}}$. There is always a function $\psi_{0}$ that minimizes the energy in the class of vector fields of type (1.6), and it satisfies (1.5). The problem is to show that this $\psi_{0}$ is a global minimizer. In terms of $f(r)$, (1.5) reads

$$
-f^{\prime \prime}-\frac{1}{r} f^{\prime}+\frac{1}{r^{2}} f+J^{\prime}\left(f^{2}\right) f=0
$$

with $f(0)=0$ and $f(1)=1$. The solution to this problem is unique [HH]. It is not hard to see that $f$ is monotone increasing, but this fact is not needed in this paper.

Although we cannot prove the full hedgehog conjecture, we are able to verify that the hedgehog is stable, that is to say that all the eigenvalues of the self-adjoint second variation operator $H$, defined by the quadratic form,

$$
\left.\frac{1}{2} \frac{\mathrm{d}^{2}}{\mathrm{~d} \varepsilon^{2}} \mathcal{E}\left(\psi_{0}+\varepsilon v\right)\right|_{\varepsilon=0}=(v, H v),
$$


are nonnegative. Specifically $H$ is given by

$$
H v=-\Delta v+J^{\prime}\left(\psi_{0}^{2}\right) v+2 J^{\prime \prime}\left(\psi_{0}^{2}\right)\left\langle\psi_{0}, v\right\rangle \psi_{0}
$$

for vector fields $v$ that vanish on $\partial \mathbf{D}$. Here $\langle a, b\rangle$ is the inner product on $\mathbf{R}^{2}$.

We believe that all the eigenvalues of $H$ are strictly positive but we cannot show this. If they are, then we can reach the following conclusion: For small $\lambda$ the hedgehog is certainly the global minimizer because $\psi \rightarrow \mathcal{E}(\psi)$ is strictly convex and hence the global minimizer is unique. If the hedgehog ceases to be the minimizer for large $\lambda$ then the non-hedgehog minimizer cannot be close to the hedgehog. In other words, a simple bifurcation away from the hedgehog cannot occur.

\section{Statements of theorems and lemmas}

The following three theorems will be proved in the next section in the order $2,3,1$. Theorem 1 is our main result. Theorem 3 will require three lemmas which we list here. Lemmas 1 and 2 on rearrangements are well known.

The proof of Theorem 2 uses some simple facts about convexity. This theorem holds for the analogous Ginzburg-Landau problem in $\mathbf{R}^{n}$ for any $n$, not just for $n=2$. Theorem 1 is a Corollary of Theorems 2 and 3 .

Theorem 1 (Weak stability of the symmetric minimizer). The eigenvalues of $H$ in $(1.8,1.9)$ (with Dirichlet boundary conditions) are all nonnegative. The complex eigenfunctions of $H$ can all be chosen to have the following form

$$
v(r, \theta)=e^{i m \theta}\left(\begin{array}{c}
a(r) e^{i \theta}+b(r) e^{-i \theta} \\
-i a(r) e^{i \theta}+i b(r) e^{-i \theta}
\end{array}\right)
$$

for suitable real functions $a=a_{m}$ and $b=b_{m}$ and with $m=0, \pm 1, \pm 2, \ldots$. Clearly, $\bar{v}$, the complex conjugate, is also an eigenvector with the same eigenvalue as $v$. The lowest eigenvalue of $H$ belongs either to $m=0$ or to $m= \pm 1$.

Remark: Both cases, $m=0$ or $m=1$, can occur-depending on $J$. When $J=0, m=1$ is optimal with $a(r)=0$. The lowest eigenfunction of $-\Delta$ is well known to be nodeless. When $J$ is very large the best choice is $m=0$ with $b(r) \simeq-a(r)$ because $a=-b$ makes $\left\langle\psi_{0}, v\right\rangle$ vanish.

Theorem 2 (Partial convexity of the energy functional $\mathcal{E}(\psi)$ ). Suppose $\psi=\left(\psi_{1}, \ldots, \psi_{n}\right)$ is a real vector field in $H^{1}\left(\mathbf{B}_{n}\right)$ that satisfies $\psi(x)=$ 
$x$ on the boundary of $\mathbf{B}_{n}$. Suppose that $\psi(x)^{2}=\sum_{i=1}^{n} \psi_{i}(x)^{2} \leq 1$ for all $x$ and suppose that each component $\psi_{i}$ satisfies

$$
\int_{\mathbf{S}^{n-1}} \psi_{i}(r \omega) \mathrm{d} \omega=0
$$

for all $r$. Define the vector field $\widetilde{\psi}(x)$ by

$$
\widetilde{\psi}(r \omega)=h(r) \omega, \quad \omega \in \mathbf{S}^{n-1}
$$

where $h$ is the spherical average of $|\psi|^{2}$, i.e.,

$$
h(r)=\left[\frac{1}{\left|\mathbf{S}^{n-1}\right|} \int_{\mathbf{S}^{n-1}}\left(\sum_{i} \psi_{i}(r \omega)^{2}\right) \mathrm{d} \omega\right]^{1 / 2}
$$

and $\left|\mathbf{S}^{n-1}\right|=\int_{\mathbf{S}^{n-1}} \mathrm{~d} \omega$. Then (with $\mathcal{E}(\psi)$ given by the obvious generalization to $\mathbf{B}_{n}$ of (1.3)),

$$
\mathcal{E}(\widetilde{\psi}) \leq \mathcal{E}(\psi)
$$

If we assume that $h(r)>0$ for all $r>0$ then equality occurs in (2.5) only if $\psi=\widetilde{\psi}$.

Theorem 3 (Rearrangements of special vector fields). Suppose that $\psi$ is a vector field in $\mathcal{C}$ and suppose that there exists some fixed vector $\omega_{0} \in \mathbf{S}^{1}$ such that

$$
\psi\left(t \omega_{0}\right)=h(t) \omega_{0}
$$

for all $t \in[-1,1]$. Then there is a vector field $\widehat{\psi} \in \mathcal{C}$ satisfying (2.6) and, additionally,

$$
\begin{aligned}
\text { (i) } \widehat{\psi}(x) & =-\widehat{\psi}(-x) \text { for all } x \in \mathbf{D}, \\
\text { (ii) } & \mathcal{E}(\widehat{\psi}) \leq \mathcal{E}(\psi)
\end{aligned}
$$

Remark: The following might help to clarify the relation between Theorems 2 and 3 . Write a minimizing $\psi \in \mathcal{C}$ in complex form as

$$
\phi(r, \theta)=\sum_{k=-\infty}^{\infty} c_{k}(r) e^{i k \theta}
$$


with $c_{k}(1)=0$ if $k \neq 1$ and $c_{1}(1)=1$. If $c_{0}(r) \equiv 0$ then Theorem 2 applies and we learn that the hedgehog is the minimizer, i.e., $c_{k}(r) \equiv 0$ for $k \neq 1$.

Next suppose that we take a $\phi$ in the form (2.9) in which only at most two of the $c_{k}$ 's are not identically zero, say $c_{1}$ and $c_{m}$ with $m \neq 1$. Then we claim that we can choose the two $c$ 's to be real functions without raising the energy. Having done this, Theorems 2 and 3 apply and we again learn that the energy minimizing choice in this restricted category has $c_{m} \equiv 0$ for $m \neq 1$. The proof of this assertion is the following. We write $c_{j}(r)=$ $p_{j}(r) \exp \left[i \alpha_{j}(r)\right]$ with $p_{j} \geq 0$ and $\alpha_{j}$ real. Then

$$
|\phi(r, \theta)|^{2}=p_{1}(r)^{2}+p_{m}(r)^{2}+2 p_{1}(r) p_{m}(r) \cos \left[(m-1) \theta+\alpha_{m}(r)-\alpha_{1}(r)\right],
$$

and we observe two things: If we replace $\alpha_{1}$ and $\alpha_{m}$ by zero then

(i) the gradient term in $\mathcal{E}$ can only decrease;

(ii) the $J$ term does not change because by a trivial shift of $\theta$, the $\theta$ integral does not depend on $\alpha_{m}(r)-\alpha_{1}(r)$. (The convexity of $J$ plays no role here.)

The lemmas about symmetric decreasing rearrangements that we shall need are the following. The first was basically proved by Chiti [CG] and then by Crandall-Tartar [CT]. For some generalizations see [AL], 2.2 and 2.3 .

Lemma 1. Let $f$ and $g$ be nonnegative functions on $\mathbf{R}^{n}$ and let $J: \mathbf{R} \rightarrow$ $\mathbf{R}^{+}$be a convex function with $J(0)=0$. Then

$$
\int_{\mathbf{R}^{n}} J\left(f^{*}(x)-g^{*}(x)\right) \mathrm{d} x \leq \int_{\mathbf{R}^{n}} J(f(x)-g(x)) \mathrm{d} x
$$

where $f^{*}$ and $g^{*}$ are the symmetric decreasing rearrangements of $f$ and $g$.

Lemma 2 (Rearrangements and gradient norms). For $u \in H_{0}^{1}([-a, a])$ define $u^{*}=|u|^{*}$. Then $u^{*} \in H_{0}^{1}([-a, a])$ and

$$
\int\left(\frac{\mathrm{d} u^{*}}{\mathrm{~d} x}\right)^{2} \leq \int\left(\frac{\mathrm{d} u}{\mathrm{~d} x}\right)^{2} .
$$

Lemma 3 (Cutting argument). Let $\psi=(f, g) \in \mathcal{C}$ and assume in addition that $g\left(x_{1}, 0\right)=0$ for $x_{1} \in[-1,1]$. Then there exists $\widetilde{\psi}=(\widetilde{f}, \widetilde{g}) \in \mathcal{C}$ such that for all $x=\left(x_{1}, x_{2}\right)$ in $\mathbf{D}$

(i) $\widetilde{g}\left(x_{1}, x_{2}\right) \geq x_{2}$ for $x_{2} \geq 0$ and $\widetilde{g}\left(x_{1}, x_{2}\right) \leq x_{2}$ for $x_{2} \leq 0$,

(ii) $|\widetilde{\psi}(x)| \leq 1$ for all $x \in \mathbf{D}$ and hence $\widetilde{f}\left(x_{1}, x_{2}\right)^{2} \leq 1-x_{2}^{2}$,

(iii) $\mathcal{E}(\widetilde{\psi}) \leq \mathcal{E}(\psi)$. 


\section{Proofs}

3A. Proof of Theorem 2. Since $\sum_{i=1}^{n} \psi_{1}(x)^{2} \leq 1$, and since $t \mapsto J(t)$ is convex we have, by Jensen's inequality, that

$$
\frac{1}{\left|\mathbf{S}^{n-1}\right|} \int_{\mathbf{S}^{n-1}} J\left(|\psi(r \omega)|^{2}\right) \mathrm{d} \omega \geq J\left(h(r)^{2}\right)
$$

and hence

$$
\int_{\mathbf{B}_{n}} J\left(|\psi(x)|^{2}\right) \mathrm{d} x \geq \int_{\mathbf{B}_{n}} J\left(h(r)^{2}\right) \mathrm{d} x=\int_{\mathbf{B}_{n}} J\left(|\widehat{\psi}(x)|^{2}\right) \mathrm{d} x .
$$

To estimate the kinetic energy we expand each component, $\psi_{j}$, into normalized spherical harmonics, $Y_{l m}$, with coefficients $c_{j}^{l m}(r)$.

$$
\psi_{j}(r \omega)=\sum_{l=1}^{\infty} \sum_{m} c_{j}^{l m}(r) Y_{l m}(\omega)
$$

Here $l$ denotes the irreducible representation of $S O(n)$, while $m$ is a multiindex that labels the rows. The reason $l=0$ is absent is that

$$
\int_{\mathbf{S}^{n-1}} \psi_{j}(r \omega) \mathrm{d} \omega=0 \text { for every } 0<r \leq 1
$$

Note that $h(r)^{2}=\sum_{j=1}^{n} \sum_{l=1}^{\infty} \sum_{m}\left(c_{j}^{l m}(r)\right)^{2} /\left|\mathbf{S}^{n-1}\right|$. It is well known that

$$
\int_{\mathbf{B}_{n}}\left|\nabla \psi_{j}\right|^{2}=\sum_{l=1}^{\infty} \sum_{m} \int_{0}^{1}\left\{\left(\mathrm{~d} c_{j}^{l m} / \mathrm{d} r\right)^{2}+\frac{l(l+n-2)}{r^{2}}\left(c_{j}^{l m}\right)^{2}\right\} r^{n-1} \mathrm{~d} r .
$$

Since $h \frac{\mathrm{d} h}{\mathrm{~d} r}=\sum_{j=1}^{n} \sum_{l=1}^{\infty} \sum_{m}\left(c_{j}^{l m} \frac{\mathrm{d} c_{j}^{l m}}{\mathrm{~d} r}\right) /\left|\mathbf{S}^{n-1}\right|$ we get, by Schwarz's inequality, that

$$
\left|\frac{\mathrm{d} h}{\mathrm{~d} r}\right|^{2} \leq \sum_{j=1}^{n} \sum_{l=1}^{\infty} \sum_{m}\left(\frac{\mathrm{d} c_{j}^{l m}}{\mathrm{~d} r}\right)^{2} /\left|\mathbf{S}^{n-1}\right|
$$

Obviously,

(3A.6)

$$
\sum_{l=1}^{\infty} \sum_{m} \int_{0}^{1} l(l+n-2)\left(c_{j}^{l m}\right)^{2} r^{n-3} \mathrm{~d} r \geq \sum_{l=1}^{\infty} \sum_{m} \int_{0}^{1}(n-1)\left(c_{j}^{l m}\right)^{2} r^{n-3} \mathrm{~d} r
$$


with equality only if $c_{j}^{l m} \equiv 0$ for all $l \geq 2$. In that case we can write

$$
\psi_{j}(x)=\sqrt{n} \sum_{k=1}^{n} \mathrm{~d}_{j}^{k}(r) \frac{x_{k}}{r}
$$

and $h(r)^{2}=\sum_{j, k=1}^{n} d_{j}^{k}(r)^{2}$. In general, by summing over $j$, we find that

$$
\int_{\mathbf{B}_{n}}|\nabla \psi|^{2} \geq\left|\mathbf{S}^{n-1}\right| \int_{0}^{1}\left\{(\mathrm{~d} h(r) / \mathrm{d} r)^{2}+\frac{n-1}{r^{2}} h(r)^{2}\right\} r^{n-1} \mathrm{~d} r=\int_{\mathbf{B}_{n}}|\nabla \widetilde{\psi}|^{2},
$$

with equality only if (3A.7) is satisfied.

In short, (2.5) has been proved and we know that equality requires (3A.7). Our final task is to show that equality in (2.5) also requires $d_{j}^{k}(r)=h(r) \delta_{k, j} / \sqrt{n}$ when $h(r)>0$ for all $r>0$.

Inequality (3A.5) was obtained by using Schwarz's inequality. In order to have equality we must have that

$$
\frac{\mathrm{d}}{\mathrm{d} r} d_{j}^{k}(r)=\lambda(r) d_{j}^{k}(r)
$$

for some function $\lambda(r)$ not depending on $j$ and $k$. By multiplying (3A.9) on both sides by $d_{j}^{k}(r)$ and summing over $k$ and $j$ we have $h(r) h^{\prime}(r)=$ $\lambda(r) h(r)^{2}$. Since $h(r)>0$ for all $r>0$ we have that

$$
\lambda(r)=h^{\prime}(r) / h(r)
$$

This function is integrable away from the origin and hence (3A.9) yields

$$
d_{j}^{k}(r)=\mu(r) d_{j}^{k}(1)
$$

with $\mu(r)=\exp \left\{-\int_{r}^{1} \lambda(s) \mathrm{d} s\right\}$. By assumption, $d_{j}^{k}(1)=n^{-1 / 2} \delta_{j, k}$, and this yields the desired conclusion with $\mu(r)=h(r)$.

3B. Proof of Theorem 3. Without loss of generality, we can assume $\omega_{0}$ $=(1,0)$. Our hypothesis is that if $\psi(x)=(f(x), g(x))$ then $g\left(x_{1}, 0\right)=0$ for $-1 \leq x_{1} \leq 1$. By Lemma 3 (cutting argument) we can assume two important facts about our $f$ and $g$ :

(i) $f\left(x_{1}, x_{2}\right)^{2} \leq 1-x_{2}^{2}$;

(ii) $g\left(x_{1}, x_{2}\right) \geq x_{2}$ if $x_{2}>0$ and $g\left(x_{1}, x_{2}\right) \leq x_{2}$ if $x_{2} \leq 0$. 
We can also assume that $f^{2}+g^{2} \leq 1$.

The first step is to define $\widetilde{g}$ in the following way. For each, fixed $x_{2}$ we replace the function $x_{1} \mapsto g\left(x_{1}, x_{2}\right)$ by its (one-dimensional) symmetric decreasing rearrangement $g^{*}\left(x_{1}, x_{2}\right)$ if $x_{2} \geq 0$ and we replace it by $-\left|g\left(x_{1}, x_{2}\right)\right|^{*}$ if $x_{2} \leq 0$. By (ii) above, $\widetilde{g}$ satisfies the correct boundary condition, (i.e., $g\left(x_{1}, x_{2}\right)=x_{2}$ on $\partial \mathbf{D}$ ), and $\widetilde{g}$ also satisfies (ii) above.

The next step, the definition of $\widetilde{f}\left(x_{1}, x_{2}\right)$, is a bit more complicated. First, let $f^{\#}$ be the symmetric increasing rearrangement of $|f|$. [Another way to say this is that $\left.1-f^{\#}=(1-|f|)^{*}\right]$. Once again, the rearrangement is done on each line $x_{2}=$ constant. We note that $f\left(x_{1}, x_{2}\right)$ is continuous in $x_{1}$ for a.e. $x_{2}$ (because it is an $H^{1}(\mathbf{R})$ function for a.e. $x_{2}$ ) and has antisymmetric boundary values at $x_{1}= \pm\left(1-x_{2}^{2}\right)^{1 / 2}$. Therefore $f^{\#}$ is a continuous function of $x_{1}$ (indeed, it is an $H^{1}(\mathbf{R})$ function by Lemma 2) and $f^{\#}\left(0, x_{2}\right)=0$. By (i) above, $f^{\#} \leq\left(1-x_{2}^{2}\right)^{1 / 2}$. Moreover, $f^{\#}=$ $\left(1-x_{2}^{2}\right)^{1 / 2}$ on $\partial \mathbf{D}$ since $x_{1} \mapsto\left|f\left(x_{1}, x_{2}\right)\right|$ is continuous and $|f|$ satisfies the same boundary condition. Now define

$$
\widetilde{f}\left(x_{1}, x_{2}\right)=\left\{\begin{aligned}
f^{\#}\left(x_{1}, x_{2}\right) & \text { if } x_{1}>0 \\
-f^{\#}\left(x_{1}, x_{2}\right) & \text { if } x_{1}<0
\end{aligned}\right.
$$

which satisfies the correct conditions on $\partial \mathbf{D}$. We also note that $\left|\partial \tilde{f} / \partial x_{1}\right|=$ $\left|\partial f^{\#} / \partial x_{1}\right|$ and $\left|\partial \tilde{f} / \partial x_{2}\right|=\left|\partial f^{\#} / \partial x_{2}\right|$.

Our task is to show that these rearrangements decrease both terms in the functional $\mathcal{E}$. We turn to the gradient norms first. By Lemma 2 we have that $\int_{\mathbf{D}}\left(\partial \widetilde{g} / \partial x_{1}\right)^{2}$ does not increase and the same is true for $\int_{\mathbf{D}}\left(\partial \widetilde{f} / \partial x_{1}\right)^{2}$. We next show that $\int_{\mathbf{D}}\left(\partial \widetilde{g} / \partial x_{2}\right)^{2}$ does not increase either. (The argument for $\tilde{f}$ is essentially identical.) There are several ways to prove this, and one way is the following. An easy approximation argument shows that

$$
\int_{\mathbf{D}}\left(\partial g / \partial x_{2}\right)^{2}=\lim _{\delta \rightarrow 0} \delta^{-2} \int_{\mathbf{D}}\left[g\left(x_{1}, x_{2}+\delta\right)-g\left(x_{1}, x_{2}\right)\right]^{2} \mathrm{~d} x_{1} \mathrm{~d} x_{2}
$$

(Here, $g\left(x_{1}, x_{2}\right)$ has to be extended to be $x_{2}$ outside D.) The result we want - that replacement of $g$ by $\widetilde{g}$ does not increase the two sides of (3B.2)follows from a trivial modification of Lemma 1.

To summarize, the vector field $\widetilde{\psi}$ is in $\mathcal{C}$ and its gradient norms are not bigger than those of $\psi$.

The penultimate step is to prove that $|\widetilde{\psi}(x)|^{2} \leq 1$ for all $x \in \mathbf{D}$. Let $K(t)=[\max (0, t)]^{2}$. Then

$$
\int_{\mathbf{D}} K\left(g^{2}-\left(1-f^{2}\right)\right)=0
$$


since $|\psi|^{2}=f^{2}+g^{2} \leq 1$. By Lemma 1, however,

$$
\int_{\mathbf{D}} K\left(g^{2}-\left(1-f^{2}\right)\right) \geq \int_{\mathbf{D}} K\left(\widetilde{g}^{2}-\left(1-\widetilde{f}^{2}\right)\right)
$$

since $\left(g^{2}\right)^{*}=\widetilde{g}^{2}$ for each line, $x_{2}=$ constant, and, similarly, $\left(1-f^{2}\right)^{*}=$ $\left(1-\widetilde{f}^{2}\right)$ since $f^{2} \leq 1$ and $\widetilde{f}^{2} \leq 1$. If $\widetilde{g}^{2}+\widetilde{f}^{2}>1$ on a set of positive measure, the right side of (3B.4) would be positive, but this is precluded by (3B.3).

Finally, we turn to the $J$ term in $\mathcal{E}$. We can define $L(t)=J(1-t)$ for $0 \leq t \leq 1$. (The definition of $L(t)$ for $t<0$ or $t>1$ is not needed since $0 \leq t \leq 1$ in our application.) Then, by Lemma 1 and the same reasoning as for $K$ above,

$$
\int_{\mathbf{D}} L\left(\left(1-f^{2}\right)-g^{2}\right) \geq \int_{\mathbf{D}} L\left(\left(1-\widetilde{f}^{2}\right)-\widetilde{g}^{2}\right)
$$

which is the same as $\int J\left(|\psi|^{2}\right) \geq \int J\left(|\widetilde{\psi}|^{2}\right)$.

Thus far we have constructed a $\widetilde{\psi}$ with $\mathcal{E}(\widetilde{\psi}) \leq \mathcal{E}(\psi)$ and with $\widetilde{f}\left(x_{1}, x_{2}\right)=$ $-\widetilde{f}\left(-x_{1}, x_{2}\right)$ and $\widetilde{g}\left(x_{1}, x_{2}\right)=\widetilde{g}\left(-x_{1}, x_{2}\right)$. The final step is to use this $\widetilde{\psi}$ to construct a $\widehat{\psi}$ satisfying $\widehat{\psi}(x)=-\widehat{\psi}(-x)$ and $\mathcal{E}(\widehat{\psi}) \leq \mathcal{E}(\widetilde{\psi})$. Let $\mathbf{D}_{+}$denote the upper hemidisc $\left\{\left(x_{1}, x_{2}\right): x_{2} \geq 0\right\} \cap \mathbf{D}$ and $\mathbf{D}_{-}$the lower hemidisc. Let $\left(\widetilde{f}_{ \pm}, \widetilde{g}_{ \pm}\right)$denote $\widetilde{\psi}$ restricted to $\mathbf{D}_{+}$and $\mathbf{D}_{-}$. Consider the following two vector fields.

$$
\begin{aligned}
& \psi_{1}= \begin{cases}\left(\tilde{f}_{+}\left(x_{1}, x_{2}\right), \widetilde{g}_{+}\left(x_{1}, x_{2}\right)\right) & \text { in } \mathbf{D}_{+} \\
\left(\tilde{f}_{+}\left(x_{1},-x_{2}\right),-\widetilde{g}_{+}\left(x_{1},-x_{2}\right)\right) & \text { in } \mathbf{D}_{-}\end{cases} \\
& \psi_{2}= \begin{cases}\left(\tilde{f}_{-}\left(x_{1},-x_{2}\right),-\widetilde{g}_{-}\left(x_{1},-x_{2}\right)\right) & \text { in } \mathbf{D}_{+} \\
\left(\tilde{f}_{-}\left(x_{1}, x_{2}\right), \widetilde{g}_{-}\left(x_{1}, x_{2}\right)\right) & \text { in } \mathbf{D}_{-}\end{cases}
\end{aligned}
$$

Clearly $\psi_{1,2}(x)=-\psi_{1,2}(-x)$. Also, $\psi_{1}$ and $\psi_{2}$ are in $\mathcal{C}$ because $\widetilde{g}\left(x_{1}, 0\right)=0$. Moreover,

$$
\mathcal{E}\left(\psi_{1}\right)+\mathcal{E}\left(\psi_{2}\right)=2 \mathcal{E}(\widetilde{\psi}) .
$$

Therefore, $\psi_{1}$ or $\psi_{2}$ is a vector field satisfying the conclusion of Theorem 3.

3C. Proof of Theorem 1. The basic fact, which we shall prove later, is that the real eigenfunction of $H$ can be chosen to have at least one of the following symmetry properties for all $x \in D$.

(a) $\quad v(x)=-v(-x)$

(b) $\quad v(x)=P v\left(P^{-1} x\right)$ 
where

$$
P=\left(\begin{array}{cc}
1 & 0 \\
0 & -1
\end{array}\right)
$$

is the reflection about the $x_{1}$-axis in $\mathbf{R}^{2}$. This is not to say that every eigenfunction has one of these properties, but we do assert that each eigenvalue of $H$ has at least one eigenfunction of type (a) or (b). Since we are interested only in the eigenvalues of $H$, we may assume (a) or (b).

Now consider $\psi_{\varepsilon}:=\psi_{0}+\varepsilon v \in \mathcal{C}$, with $v$ as in (3C.1). In case (a), $\psi_{\varepsilon}(x)=-\psi_{\varepsilon}(-x)$ for all $\varepsilon$. In case (b), $\psi_{\varepsilon}$ satisfies hypothesis (2.6) of Theorem 3, with $\omega_{0}=(1,0)$. By Theorem 2, in case (a) there is a $\widetilde{\psi}_{\varepsilon}$ with $\mathcal{E}\left(\widetilde{\psi}_{\varepsilon}\right) \leq \mathcal{E}\left(\psi_{\varepsilon}\right)$ and $\widetilde{\psi}_{\varepsilon}$ is a hedgehog (1.6). Thus,

$$
\mathcal{E}\left(\psi_{\varepsilon}\right) \geq \mathcal{E}\left(\widetilde{\psi}_{\varepsilon}\right) \geq \mathcal{E}\left(\psi_{0}\right)
$$

since $\psi_{0}$ is the energy minimizer among all hedgehogs. In case (b) we first have to use Theorem 3 to obtain an intermediate $\widehat{\psi}$ that satisfies the hypothesis of Theorem 2. Again, (3C.2) holds. Since

$$
\mathcal{E}\left(\psi_{\varepsilon}\right)=\mathcal{E}\left(\psi_{0}\right)+\varepsilon^{2} \gamma \int_{\mathbf{D}} v^{2}+o\left(\varepsilon^{2}\right)
$$

where $\gamma$ is the eigenvalue of $H$ belonging to $v$, we see from (3C.2) that $\gamma \geq 0$.

There are two ways to derive the symmetry (3C.1) and we shall give both. The first is a fairly general argument and the second involves a detailed study of the eigenfunctions leading to (2.1).

General argument: Let $P$ denote reflection about some axis through the origin. For any eigenfunction, $w$, its reflection, $\left(P^{*} w\right)(x):=P w\left(P^{-1} x\right)$, is also an eigenfunction with the same eigenvalue. If $v(x)=w(x)+$ $\left(P^{*} w\right)(x)$ is not identically zero for some $P$ then $v$ is an eigenfunction satisfying (3C.1) (b). If $v$ vanishes identically for all reflections $P$ we claim that $w$ must be of type (3C.1)(a). To see this recall that any rotation $\mathcal{R}$ is the product of two reflections and hence $\mathcal{R} w\left(\mathcal{R}^{-1} x\right)=w(x)$, i.e., $w$ is rotationally symmetric. It is easy to see that $w$ must then satisfy $w(x)=k(r)\left(-x_{2}, x_{1}\right)$ for some function $k$, and hence $w$ satisfies (3C.1)(a).

Details of eigenfunctions: Let $\mathcal{R}_{\alpha}$ be the rotation through the angle $\alpha$ and let $U_{\alpha}$ be its representation given by

$$
\left(U_{\alpha} v\right)(x)=\mathcal{R}_{\alpha} v\left(\mathcal{R}_{-\alpha} x\right) .
$$

$U_{\alpha}$ is a strongly continuous one-parameter subgroup of the unitary group of $L^{2}\left(\mathbf{D} ; \mathbf{C}^{2}\right)$ and it commutes with $H$. Its infinitesimal generator is

$$
L=i \frac{\partial}{\partial \theta}+i\left(\begin{array}{cc}
0 & 1 \\
-1 & 0
\end{array}\right) \text {. }
$$


By standard arguments we can choose the eigenfunctions of $H$ to be eigenfunctions of $L$. By solving $L v=\nu v$ we find that $v(\theta)$ must be of the form (2.1). $\nu$ must be an integer since $v(0)=v(2 \pi)$. Furthermore, a glance at the eigenvalue equation reveals that $a$ and $b$ can be taken to be real.

Next we verify that the lowest eigenvalue belongs to $m=0$ or $m= \pm 1$. Suppose, on the contrary, that the lowest one belongs to $M>1$ ( $m$ and $-m$ are the same by complex conjugation). Then define a comparison function by $\widehat{v}:=e^{-i \theta} v$. Obviously, the $J$-term is unchanged. The only term that changes in the gradient norm is the replacement of $I:=\int_{\mathbf{D}}\left|\frac{\partial}{\partial \theta} v\right|^{2} r^{-2}$ by $\widehat{I}:=\int_{\mathbf{D}}\left|\frac{\partial}{\partial \theta} e^{-i \theta} v\right|^{2} r^{-2}$. One easily computes that

$$
I=2 \int_{\mathbf{D}}\left\{(M+1)^{2} a(r)^{2}+(M-1)^{2} b(r)^{2}\right\} r^{-2}
$$

and hence $\widehat{I}<I$ if $M>1$.

Now consider (2.1) with $m=0$. This vector $v$ satisfies $v(x)=-v(-x)$ (because changing $x$ to $-x$ amounts to changing $\theta$ to $\theta+\pi$ ). Thus, all $m=0$ eigenfunctions satisfy (3C.1)(a). Indeed all even- $m$ eigenfunctions have this property.

When $m=1$ we claim that (3C.1)(b) holds - thus completing our proof. Take the real part of $(2.1)$, which is $v(r, \theta)=(a(r) \cos 2 \theta+b(r), a(r) \sin 2 \theta)$; this satisfies (3C.1)(b) with $P$ being reflection about the $x_{1}$-axis.

3D. Remarks on Lemma 1. The Chiti, Crandall-Tartar theorem requires the convex function $J$ to be even. It is usually stated as

$$
J: \mathbf{R}^{+} \rightarrow \mathbf{R}^{+}, \quad J(0)=0
$$

and with (2.10) replaced by

$$
\int_{\mathbf{R}^{n}} J\left(\left|f^{*}-g^{*}\right|\right) \leq \int_{\mathbf{R}^{n}} J(|f-g|) .
$$

It is a simple matter to derive (2.10) from (2.10a) but we are unaware of (2.10) in the literature. Evidently, it suffices to prove (2.10) for the extremal convex functions, i.e., $J$ of the type $J(t)=\lambda(t-a)_{+}$or $J(t)=\lambda(t+a)_{-}$ with $t_{ \pm}:=\max \{ \pm t, 0\}$ and $a, \lambda \geq 0$. Consider $\lambda(t-a)_{+}$. By replacing $f$ by $(f-a)_{+}$we may as well take $a=0$ and $\lambda=2$. Then $2 t_{+}=|t|+t$. We can assume $f-g \in L^{1}(\mathbf{R})$, for otherwise the right side of $(2.10)$ is $+\infty$. It is easy to see that $f^{*}-g^{*} \in L^{1}(\mathbf{R})$ as well. Then, since $2 t_{+}=|t|+t$, (2.10) reads

$$
\int\left\{\left|f^{*}-g^{*}\right|-|f-g|\right\} \geq \int\left\{f-g+g^{*}-f^{*}\right\} .
$$

Indeed, the left side is nonnegative by (2.10a) and the right side is zero since $f, f^{*}$ and $g, g^{*}$ are equimeasurable. 
3E. Remarks on Lemma 2. (2.11) also holds for functions on $\mathbf{R}^{n}$, but we need only the $\mathbf{R}^{1}$ version. For some historical remarks about this inequality see [AL] 1.1, 2.6 and 2.7. There are many proofs of it but the simplest (in our view) is in [LE1], Lemma 5. The method of [LE1], Lemma 5 also proves a generalization that would suffice for our needs in the proof of Theorem 3 above. The generalization is that if $f: \mathbf{R}^{n+m} \rightarrow \mathbf{R}^{+}$and if $f^{*}: \mathbf{R}^{n+m} \rightarrow \mathbf{R}^{+}$is the symmetric decreasing rearrangement with respect to the first $n$ variables only, then

$$
\int_{\mathbf{R}^{n+m}}\left|\nabla f^{*}\right|^{2} \leq \int_{\mathbf{R}^{n+m}}|\nabla f|^{2}
$$

3F. Proof of Lemma 3. We shall construct $\widetilde{\psi}$ by a sequence of steps.

Step 1. $\quad \psi \mapsto T_{1} \psi= \begin{cases}\psi & \text { if }|\psi| \leq 1 \\ \psi /|\psi| & \text { if }|\psi| \geq 1\end{cases}$

[with $\left.|\psi|=\left(f^{2}+g^{2}\right)^{1 / 2}\right]$. An easy exercise shows that $\left\|\nabla T_{1} \psi\right\|_{2} \leq\|\nabla \psi\|_{2}$. Furthermore, $\int J\left(|\psi|^{2}\right) \geq \int J\left(\left|T_{1} \psi\right|^{2}\right)$ because $J(t) \geq 0$ when $t>1$ while $J(1)=0$. Therefore, without loss of generality we can assume that $|\psi(x)| \leq$ 1 for all $x$.

Step 2.

$$
\psi \rightarrow T_{2} \psi=(f, h) \quad \text { with } h\left(x_{1}, x_{2}\right)= \begin{cases}\max \left\{x_{2}, g\left(x_{1}, x_{2}\right)\right\} & \text { if } x_{2} \geq 0 \\ \min \left\{x_{2}, g\left(x_{1}, x_{2}\right)\right\} & \text { if } x_{2} \leq 0 .\end{cases}
$$

Obviously $\left|T_{2} \psi(x)\right| \geq|\psi(x)|$ for all $x$. The condition $g\left(x_{1}, 0\right)=0$ guarantees that $T_{2} \psi \in \mathcal{C}$.

Step $3 . \quad \psi \mapsto T_{3} \psi=T_{1} T_{2} \psi$.

If we write $T_{1} T_{2} \psi=(\widehat{f}, \widehat{g})=\widehat{\psi}$ we can easily verify the following for all $x$ (using $|\psi| \leq 1$ )

$$
\begin{aligned}
1 & \geq\left|T_{3} \psi(x)\right| \geq|\psi(x)| \\
|\widehat{f}(x)| & \leq|f(x)| \text { and } \operatorname{sgn} \widehat{f}(x)=\operatorname{sgn} f(x) \\
|\widehat{g}(x)| & \geq|g(x)| \text { and } \operatorname{sgn} \widehat{g}(x)=\operatorname{sgn} x_{2} \\
\widehat{g}(x)^{2}-g(x)^{2} & \geq \frac{1-x_{2}^{2}}{1+x_{2}^{2}}\left[x_{2}^{2}-g(x)^{2}\right]_{+} \cdot
\end{aligned}
$$

(a) is obvious because $T_{2}$ does not decrease $|\psi|$ and $T_{1}$ only cuts off $\left|T_{2} \psi\right|$ at 1 ; but $|\psi| \leq 1$ everywhere. (b) is also obvious because $T_{2}$ leaves $f$ 
invariant and $T_{1}$ can only decrease $|f|$. (c) follows from the facts that $T_{2}$ increases $|g|$, the map $t \mapsto t /\left(f^{2}+t\right)$ is monotone increasing for $t>0$, and $g^{2} \leq g^{2} /\left(f^{2}+g^{2}\right)$ since $f^{2}+g^{2} \leq 1$. Indeed, (d) gives a more quantitative estimate. To prove $(\mathrm{d})$ we recall that $T_{2} \psi=:(f, h)$. If $f^{2}+h^{2} \leq 1$ and $x_{2}^{2} \geq g^{2}$ then $|\widehat{g}|=|h|=\left|x_{2}\right|$ and (d) is certainly true. If $f^{2}+h^{2}>1$ and $x_{2}^{2} \geq g^{2}$ then

$$
\begin{aligned}
\widehat{g}^{2}-g^{2} & =\frac{x_{2}^{2}}{f^{2}+x_{2}^{2}}-g^{2} \\
& \geq \frac{x_{2}^{2}}{1-g^{2}+x_{2}^{2}}-g^{2} \\
& =\frac{1-g^{2}}{1-g^{2}+x_{2}^{2}}\left[x_{2}^{2}-g^{2}\right] \\
& \geq \frac{1-x_{2}^{2}}{1+x_{2}^{2}}\left[x_{2}^{2}-g^{2}\right] .
\end{aligned}
$$

We claim that $\mathcal{E}\left(T_{3} \psi\right) \leq \mathcal{E}(\psi)$. As far as the gradient term is concerned, $T_{2}$ replaces $g$ by the harmonic function $x_{2}$ on the set where $|g| \leq\left|x_{2}\right|$. This certainly lowers the gradient term. The $J$ term does not increase by property (a) above, since $J(t)$ is decreasing for $0 \leq t \leq 1$.

Now we iterate $T_{3}$ and denote $\left(f_{n}, g_{n}\right)=\psi_{n}:=T_{3}^{n} \psi$. By (b) and (c) $f_{n}$ and $g_{n}$ are bounded monotone sequences and converge pointwise to limit functions $\widetilde{f}$ and $\widetilde{g}$. Since $\mathcal{E}(\psi)$ is weakly lower semicontinuous we have that $\mathcal{E}(\widetilde{\psi}) \leq \mathcal{E}(\psi)$, where $\widetilde{\psi}=(\widetilde{f}, \widetilde{g})$. It is clear that $\widetilde{\psi}$ satisfies the correct boundary conditions and hence is in $\mathcal{C}$.

The only thing left to check is that $\widetilde{g}(x)^{2} \geq x_{2}^{2}$. If we define $a_{n}(x)=\left[x_{2}^{2}-\right.$ $\left.g_{n}(x)^{2}\right]_{+}$property (d) can be rewritten as $a_{n+1}(x) \leq a_{n}(x)\left(2 x_{2}^{2} /\left(1+x_{2}^{2}\right)\right)$, which shows that $a_{n}(x)$ converges to zero pointwise for all $x \in \mathbf{D}$.

\section{Acknowledgements}

We thank László Erdős for many valuable discussions.

\section{References}

[AL] F.J. Almgren, Jr. and E.H. Lieb, Symmetric decreasing rearrangement is sometimes continuous, J. Amer. Math. Soc. 2 (1989), 683-773.

[BBH] F. Bethuel, H. Brezis and F. Hélein, Ginzburg-Landau Vortices, Birkhäuser, 1994.

[CG] G. Chiti, Rearrangements of functions and convergence in Orlicz spaces, Appl. Anal. 9 (1979), 23-27. 
[CT] M.G. Crandall and L. Tartar, Some relations between nonexpansive and order preserving mappings, Proc. Amer. Math. Soc. 78 (1980), 358-390.

[HH] R.M. Hervé and M. Hervé, Etude qualitative des solutions réeles de l'équation differentielle ... (to appear).

[JT] A. Jaffe and C. Taubes, Vortices and Monopoles, Birkhäuser, 1980.

[LE1] E.H. Lieb, Existence and uniqueness of the minimizing solution of Choquard's non-linear equation, Stud. Appl. Math. 57 (1977), 93-105.

[LE2] - Remarks on the Skyrme Model, Proc. Amer. Math. Soc., Symposia in Pure Math. 54 (1993), 379-384, (Proceedings of Summer Research Institute on Differential Geometry at UCLA, July 8-28, 1990).

Department of Mathematics,Princeton University, P.O. B ox 708 , PrinceTON, N J 08544-0708

E-mail address: lieb@math.princeton.edu

School of M athematics, Georgia Institute of Technology, Athanta, Ga $30332-0160$

E-mail address: loss@math.gatech.edu 